\title{
CUTL1 induces epithelial-mesenchymal transition in non-small cell lung cancer
}

\author{
JUNFENG WANG, YANBO WANG, DAWEI SUN, FENGHAI REN, SAINAN PANG and SHIDONG XU
}

Department of Thoracic Surgery, Harbin Medical University Cancer Hospital, Harbin, Heilongjiang 150081, P.R. China

Received October 22, 2016; Accepted March 7, 2017

DOI: $10.3892 /$ or.2017.5571

\begin{abstract}
The homeobox transcription factor CUTL1 has been associated with cellular proliferation and cell cycle progression, and CUTL1 functions as an oncogene. The aim of the present study was to investigate whether CUTL1 participates in epithelial-mesenchymal transition (EMT). The expression levels of CUTL1, E-cadherin, N-cadherin and Snail were determined by immunohistochemistry assay, immunofluorescence assay or real-time quantitative reverse transcription PCR. Their roles in non-small cell lung cancer (NSCLC) were assessed by functional analyses. Protein expression was detected by western blot analysis. The CUTL1 expression levels are higher in non-small cell lung cancer (NSCLC) tissues. High CUTL1 expression in NSCLC is associated with the mesenchymal-like phenotype. Mechanistically, CUTL1 upregulates transforming growth factor $\beta$ receptor I (T $\beta R-I)$ expression, and the T $\beta$ R-I inhibitor SB431542 abolishes EMT elicited by ectopic CUTL1 expression. Transforming growth factor $\beta$ (TGF- $\beta$ ) signaling is essential for CUTL1-induced EMT in NSCLC cells. CUTL1 is downstream of TGF- $\beta$ signaling and CUTL1 is involved in the expression of the T $\beta$ R-I. This study indicates that CUTL1 may be a potential target for anti-lung cancer therapy.
\end{abstract}

\section{Introduction}

Lung cancer is the leading cause of cancer mortality worldwide (1), and non-small cell lung cancer (NSCLC) is the most common type of lung cancer. Approximately $85 \%$ of lung cancers are NSCLC, and the subtypes of NSCLC include squamous cell carcinoma, adenocarcinoma and large cell carcinoma. When diagnosed, most NSCLC patients are at an advanced and inoperable stage and therefore have limited therapeutic options (2). Moreover, the metastatic phenotype

Correspondence to: Dr Shidong Xu, Department of Thoracic Surgery, Harbin Medical University Cancer Hospital, Harbin, Heilongjiang 150081, P.R. China

E-mail: xusd163@163.com

Key words: CUTL1, epithelial-mesenchymal transition, non-small cell lung cancer, transforming growth factor $\beta$ is a major cause of lung cancer mortality. Therefore, earlier detection and prevention of metastasis is very important to prevent the progression of NSCLC (3).

The homeobox transcription factor CUTL1, also known as CutX1 or CCAAT displacement protein (cux/CDP), plays a key role in the control of normal embryonic development, differentiation and cell growth (4). CUTL1 is known as a transcriptional activator or as a transcriptional repressor $(5,6)$. CUTL1 is associated with cellular proliferation and cell cycle progression $(7,8)$, and it functions as an oncogene because its increased expression promotes the growth and migration of cancer cells (9-11). Epithelial-mesenchymal transition (EMT) is a key process that facilitates cell invasion and metastasis and is characterized by a convergent loss or relocalization of epithelial markers such as E-cadherin and $\beta$-catenin and gain of mesenchymal markers such as N-cadherin and vimentin (12). Accumulating evidence suggests that EMT is important for the initial step of tumor metastasis $(13,14)$; however, whether CUTL1 participates in EMT is currently unknown.

\section{Materials and methods}

Ethics statement. Patient information and samples were obtained with written informed consent. Each patient in the study gave written informed consent to publish the case details. The study was approved by the ethics committee of Harbin Medical University Cancer Hospital.

Cell lines, cell culture and transfection. The human NSCLC lines (H292, H358, H322, A549, Calu1, Calu6 and H23) and human embryonic kidney cell line 293T were purchased from the American Type Culture Collection (ATCC; Manassas, VA, USA) and maintained in Dulbecco's Modified Eagle's medium (DMEM) supplemented with $10 \%$ fetal bovine serum (FBS; Invitrogen, Carlsbad, CA, USA) containing 100 units/ml penicillin and 100 units/ml streptomycin (Sigma-Aldrich, St. Louis, $\mathrm{MO}, \mathrm{USA}$ ) at $37^{\circ} \mathrm{C}$ with $5 \% \mathrm{CO}_{2}$. A549 and $\mathrm{H} 322$ cells were transfected using X-tremeGENE (Roche Applied Science, Indianapolis, IN, USA) and HEK293T cells were transfected using Lipofectamine 2000 (Invitrogen) according to the manufacturer's directions. Human CUTL1 siRNA (sc-35051; Santa Cruz Biotechnology, Santa Cruz, CA, USA) was transfected into cells for $24 \mathrm{~h}$ using Lipofectamine RNAiMAX reagent (Invitrogen). T $\beta$ R-I inhibitor SB431542 (10 $\mu \mathrm{M})$ was from R\&D Systems (Minneapolis, MN, USA). 
Western blot analysis. Total cellular proteins were extracted using lysis buffer and $40 \mu \mathrm{g}$ of total proteins was used for western blot analysis. The polyvinylidene difluoride membranes were probed with antibodies against CUTL1 (1:1,000 dilution) (Novus Biologicals, Littleton, CO, USA); E-cadherin (1:1,000 dilution), N-cadherin (1:1,000 dilution), Snail (1:1,000 dilution), p-Smad3 (1:1,000 dilution) (Cell Signaling Technology, Danvers, MA, USA); or GAPDH (1:1,000 dilution). The blots were subsequently developed using the enhanced chemiluminescence method (Millipore, Billerica, MA, USA). The GAPDH signal was used as a control.

Transcription reporter assay. Cells were treated with or without $2 \mathrm{ng} / \mathrm{ml}$ TGF- $\beta 120 \mathrm{~h}$ after transfection. The cells were then harvested and analyzed with the Dual-luciferase reporter assay system (Promega, Madison, WI, USA). All assays were performed in triplicate, and all values were normalized for transfection efficiency against Renilla luciferase activities.

Real-time RT-PCR ( $q R T-P C R)$. Total RNAs were obtained using the TRIzol reagent (Invitrogen). Reverse transcription of the RNA was performed using the ImProm-II reverse transcription system (Promega) according to the manufacturer's instructions. Quantitative reverse transcriptase (qRT)-PCR was performed using an ABI PRISM 7500 sequence detector system (Applied Biosystems, Foster City, CA, USA) with gene-specific primers.

Immunofluorescence. A549 and Calu1 cells were cultured in a 12-well plate and transfected with siCUTL1. The cells were then fixed with $4 \%$ paraformaldehyde, permeabilized with $0.1 \%$ Triton $\mathrm{X}-100$, and incubated with primary antibody against E-cadherin or $\mathrm{N}$-cadherin for $1 \mathrm{~h}$ at $37^{\circ} \mathrm{C}$. The cells were then incubated with Alexa Fluor 488 for $30 \mathrm{~min}$ at $37^{\circ} \mathrm{C}$. The fluorescent images were captured using a confocal laserscanning microscope.

Immunohistochemistry. Tissue specimens were embedded in paraffin. The sections were then deparaffinized in xylene and rehydrated in an ethanol gradient. After antigen retrieval, the sections were treated with $3 \% \mathrm{H}_{2} \mathrm{O}_{2}$ for $10 \mathrm{~min}$ followed by $5 \%$ bovine serum albumin (BSA) for $30 \mathrm{~min}$. Then, the sections were incubated with primary antibodies against CUTL1 (1:200 dilution), E-cadherin (1:100 dilution), N-cadherin (1:100 dilution) or Snail (1:100 dilution) overnight at $4^{\circ} \mathrm{C}$. Visualization of antibody binding was performed using DAB staining. The nuclei were stained with hematoxylin, and the immunostaining results were independently assessed by two pathologists. The percentage of the positive cancer cells: 0 , no positive cells; 1 , $<10 \%$ positive cells; $2,10-50 \%$ positive cancer cells; $3,51-75 \%$ positive cancer cells; and $4 \geq 75 \%$ positive cancer cells. The staining intensity: 1 , no staining; 2 , weak staining; 3 , moderate staining; and 4, strong staining. The final score was equal to the area score and the intensity score. A final staining score $\geq 4$ was considered overexpression, and score $<4$ as not overexpressed.

Patients. Lung cancer specimens $(\mathrm{n}=57)$ were collected from patients with NSCLC at Harbin Medical University Cancer
Table I. Expression of CUTL1 in NSCLC tissues and their adjacent normal tissues.

\section{CUTL1}

\begin{tabular}{lccc} 
Variables & $\mathrm{n}$ & Overexpression, n $(\%)^{\mathrm{a}}$ & P-value \\
\hline Cancer tissue & 57 & $40(70.18$ & $<0.001$ \\
Adjacent tissue & 39 & $12(30.77)$ & \\
\hline
\end{tabular}

${ }^{a}$ A final staining score $\geq 4$ overexpression, and a final staining score $<4$ not overexpressed.

Hospital from 2009 to 2012 . The tissues were stored at $-80^{\circ} \mathrm{C}$ until use. All samples were from patients who had not undergone preoperative radiotherapy or chemotherapy.

The pathological staging of the 57 tumors was performed according to the tumor node metastasis (TNM) staging system.

Cell migration and invasion assay. Migration assays were performed with $8-\mu \mathrm{m}$ filters (BD Biosciences, San Jose, CA, USA). Each well was loaded with $\sim 1 \times 10^{5}$ cells. After incubation for $20 \mathrm{~h}$, the cells passing through the filter into the bottom wells were fixed in formalin and stained with crystal violet. The cells in 10 randomly selected fields (x200) from each well were counted. Transwell invasion assays were performed under the same conditions as the Transwell migration assays, but before the invasion assays, the polycarbonate filter was coated with Matrigel.

Statistical analyses. All statistical analyses were performed using SPSS 17.0 software. The $\chi^{2}$ test was used to analyze the expression of CUTL1 in NSCLC tissues and their adjacent normal tissues. Other statistical analyses were performed by Student's t-test. The data are shown as the mean \pm SD from 3 independent assays. A statistically significant difference was considered at $\mathrm{P}<0.05$.

\section{Results}

The CUTL1 expression levels are higher in clinical NSCLC tissues. To explore the roles of CUTL1 in lung cancer, we first tested the CUTL1 expression in NSCLC tissues and their corresponding normal tissues by IHC assays. The results are summarized in Table I. Specimens overexpressing CUTL1 accounted for $70.18 \%$ of the cancer samples, and most of these samples exhibited intermediate to strong staining. However, specimens overexpressing CUTL1 accounted for only $30.77 \%$ of the adjacent normal lung tissues and most of the samples exhibited weak to moderate staining. The expression of CUTL1 was significantly higher in the NSCLC specimens than in the normal lung tissues (Fig. 1A). CUTL1 expression was mainly localized in the nucleus of the NSCLC tumor cells (Fig. 1A). To study the roles of CUTL1 in metastasis, we investigated the role of CUTL1 in EMT. First, E-cadherin, $\mathrm{N}$-cadherin and Snail were detected in the lung cancer tissues and in their corresponding normal lung tissues using IHC assays (Fig. 1A). The expression of E-cadherin decreased in the cancer tissues compared to their adjacent normal 

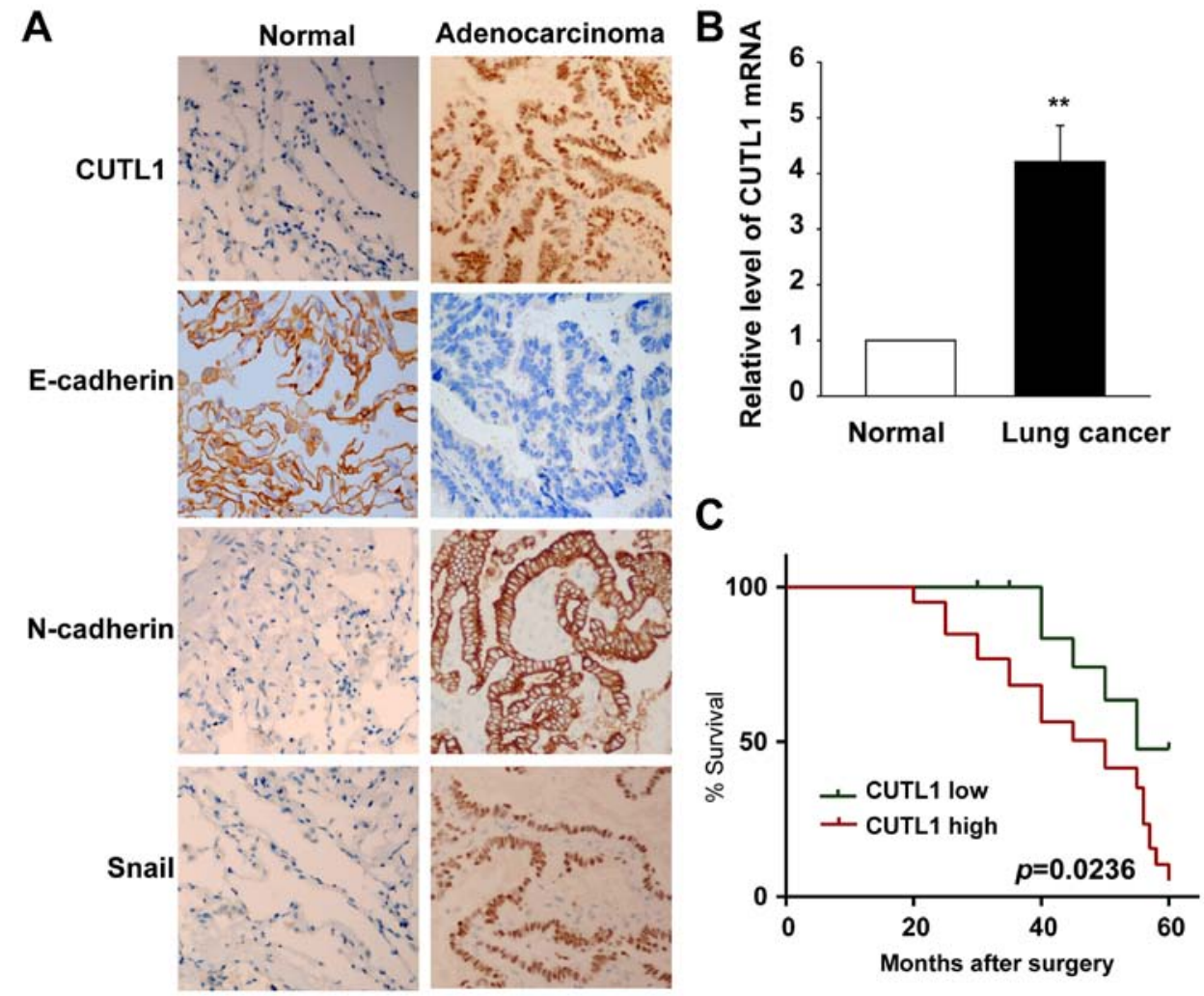

Figure 1. The expression of CUTL1, E-cadherin, N-cadherin and Snail in normal lung tissues and lung cancer tissues. (A) Immunohistochemistrical analysis of CUTL1, E-cadherin, N-cadherin and Snail (magnification, x400). (B) Real-time PCR analysis of CUTL1 mRNA. The level of CUTL1 mRNA was significantly higher in the lung cancer tissues than in the normal lung tissues. ${ }^{* *} \mathrm{P}<0.01$. (C) Kaplan-Meier survival curves for NSCLC patients with CUTL1 higher or lower expression. The difference in postoperative survival between NSCLC patients with CUTL1 higher expression and with CUTL1 lower expression was significant $(\mathrm{P}=0.0236$ by log-rank test).

tissues, but the expression of N-cadherin and Snail increased in the cancer tissues compared with their adjacent normal tissues (Fig. 1A). The levels of CUTL1 in 8 lung cancer tissues and their corresponding normal lung tissues were further detected using real-time PCR analyses. The results showed that CUTL1 mRNA was increased in the lung cancer tissues (Fig. 1B). The Kaplan-Meier survival curves showed that patients with CUTL1 higher expression were at a notably greater risk of an earlier death than those with CUTL1 lower expression $(\mathrm{P}=0.0236)$ (Fig. 1C).

High CUTL1 expression in NSCLC is associated with its mesenchymal-like phenotype. To establish the relationship between CUTL1 expression and the NSCLC cell phenotype, a panel of human NSCLC cell lines was screened for CUTL1 expression. CUTL1 was highly expressed in cell lines that were characterized as mesenchymal-like cell lines (Fig. 2A). In contrast, epithelial-like cells almost completely lacked CUTL1 (Fig. 2A).

The cellular migratory behavior of the A549 cells were studied using migration and invasion assays. Compared to the control group, fewer A549 cells passed through the Matrigel after depletion of CUTL1 (Fig. 2B). Consistently, there were fewer A549 cells that migrated when CUTL1 was downregulated (Fig. 2C), but the number of migrated H322 cells was significantly increased after the overexpression of CUTL1 (Fig. 2D and E). In A549 cells, CUTL1 knockdown resulted in a morphological switch from a migratory, mesenchymal-like phenotype to a resting, epithelium-like phenotype (Fig. 2F). These data suggest that CUTL1 is involved in an EMT-like switch and facilitates cell migration in vitro.

Effects of CUTL1 on EMT in NSCLC cell lines. To further confirm the effects of CUTL1 on EMT, we detected the expression of CUTL1, E-cadherin, N-cadherin and Snail using western blot analysis. The expression of CUTL1, N-cadherin and Snail increased, whereas the expression of E-cadherin decreased in $\mathrm{H} 322$ and $\mathrm{H} 292$ cells transfected with the pcDNA3-HA-CUTL1 plasmid (Fig. 3A). Moreover, the A549 and Calu1 cells exhibited lower levels of CUTL1, N-cadherin and Snail proteins and higher levels of the E-cadherin protein when transfected with CUTL1 siRNA (Fig. 3B). Finally, we determined the expression of E-cadherin and $\mathrm{N}$-cadherin by immunofluorescence staining. As shown in Fig. 3C and D, treatment with siCUTL1 significantly increased E-cadherin expression and decreased $\mathrm{N}$-cadherin expression in A549 and Calu1 cells. These data suggest that CUTL1 may induce EMT in NSCLC cell lines.

CUTL1 induces EMT through the TGF- $\beta$ signaling pathway. It is well known that the TG-F $\beta$ signaling pathway plays a key role in EMT (15-17). To test whether CUTL1 regulation of EMT is associated with the TGF- $\beta$ signaling pathway, we overexpressed CUTL1 in H322 cells and then treated them with the TRR-I inhibitor SB431542. The morphology of the 
A
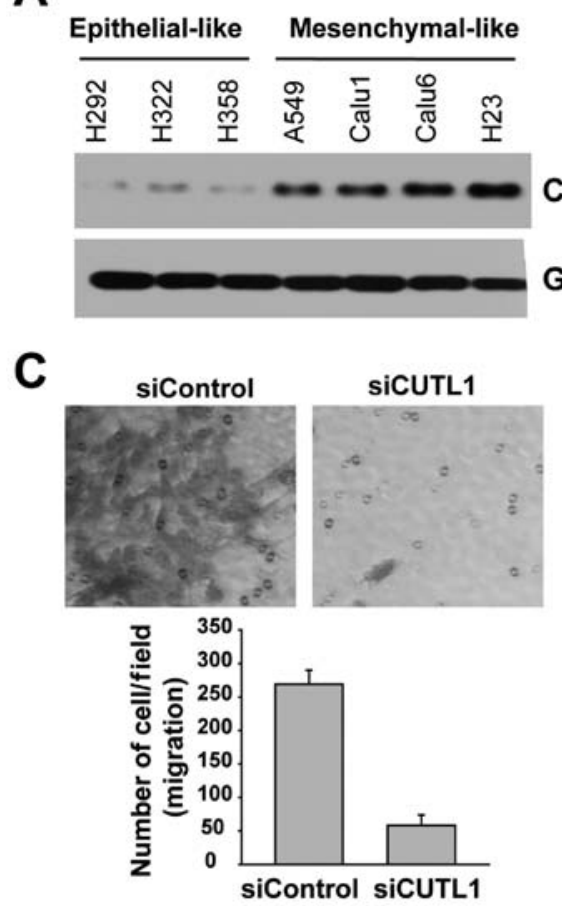

E
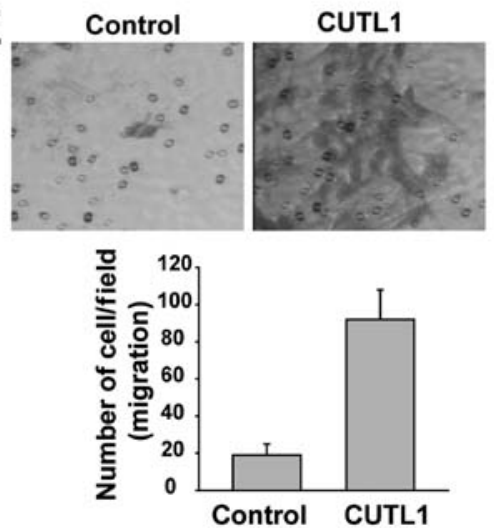

B

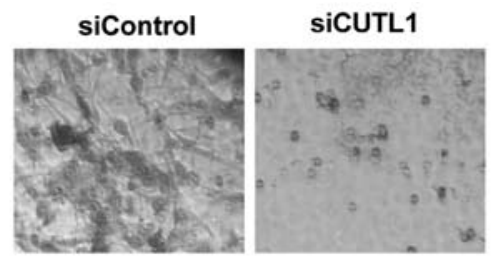

GAPDH

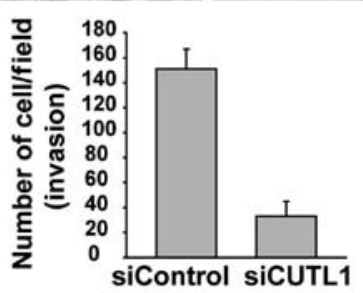

D
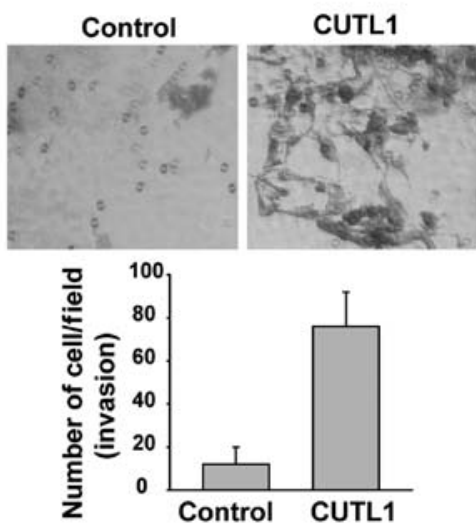

F

siControl

siCUTL1
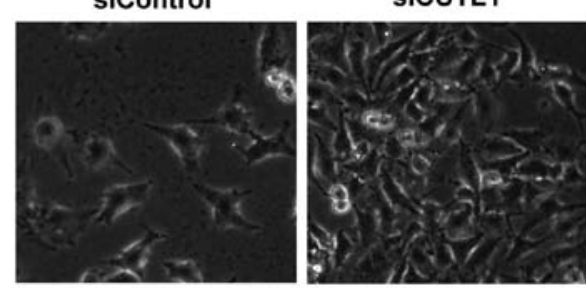

Figure 2. High CUTL1 expression in NSCLC is associated with its mesenchymal-like phenotype. (A) Western blot analysis of the CUTL1 protein expression in a panel of human NSCLC cell lines. (B) Upper panel: Transwell invasion analysis (magnification, x200); lower panel: the number of invaded A549 cells. (C) Upper panel: Transwell migration analysis; lower panel: the number of migrated A549 cells. (D) Upper panel: Transwell invasion analysis; lowe panel: the number of invaded $\mathrm{H} 322$ cells. (E) Upper panel: Transwell migration analysis; lower panel: the number of migrated H322 cells. (F) Phase-contrast microscopy shows the cell morphological changes in siCUTL1 A549 cells.

control groups treated with or without SB431542 remained unchanged (Fig. 4A). In contrast, the mesenchymal-like morphology of CUTL1-overexpressing H322 cells was reverted back to epithelial-like morphology upon treatment with SB431542 (Fig. 4A). Western blot analysis further showed that SB431542 treatment restored E-cadherin expression and abolished N-cadherin expression in CUTL1-overexpressing H322 and H292 cells (Fig. 4B). Moreover, TGF- $\beta$-mediated E-cadherin and $\mathrm{N}$-cadherin expression at the protein level was inhibited in A549 and Calu1 cells following CUTL1 depletion. These data suggest that CUTL1-induced EMT depends on the TGF- $\beta$ signaling pathway.

CUTL1 promotes T $\beta R$-I expression to positively regulate the TGF- $\beta$ signaling pathway. To identify the TGF- $\beta$ signaling pathway component that is critical for CUTL-mediated EMT, we analyzed the mRNA levels of T $\beta$ R-I, T $\beta$ R-II and T $\beta R$-III in
H322 cells ectopically expressing CUTL. Among the T $\beta$ Rs, the amount of T $\beta$ R-I increased $>7$-fold in H322 cells upon ectopic CUTL1 expression, but no significant change was detected for T $\beta$ R-II or T $\beta$ R-III (Fig. 5A). CUTL1 knockdown in A549 cells impaired Smad3 phosphorylation in response to TGF- $\beta$ exposure (Fig. 5B). In addition, knockdown of CUTL1 in A549 cells resulted in reduced activity of TGF- $\beta / \mathrm{Smad} 3$-driven SBE4-luciferase transcriptional reporter activity (Fig. 5C). These results indicate that CUTL promotes T $\beta$ R-I expression to positively regulate the TGF- $\beta$ signaling pathway in lung cancer cell lines.

\section{Discussion}

The homeobox transcription factor CUTL1 is evolutionarily highly conserved and known to regulate cell growth and differentiation in mammals $(4,5)$. CUTL1 has been shown to 
A
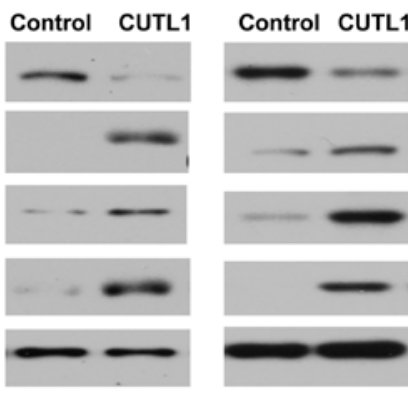

Snail

CUTL1

GAPDH

B

siControl siCUTL1 siControl siCUTL1
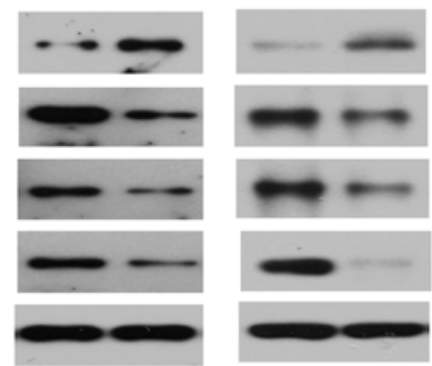

C

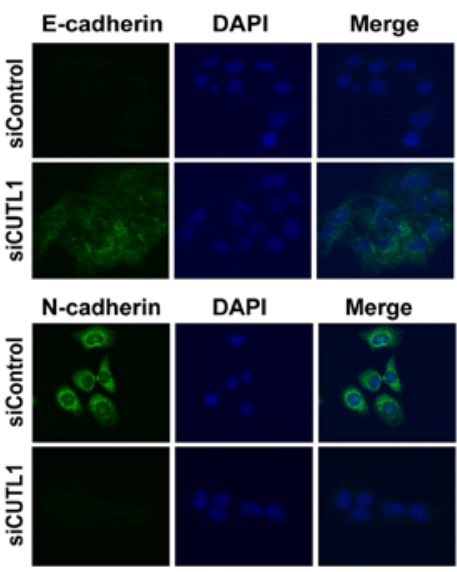

D

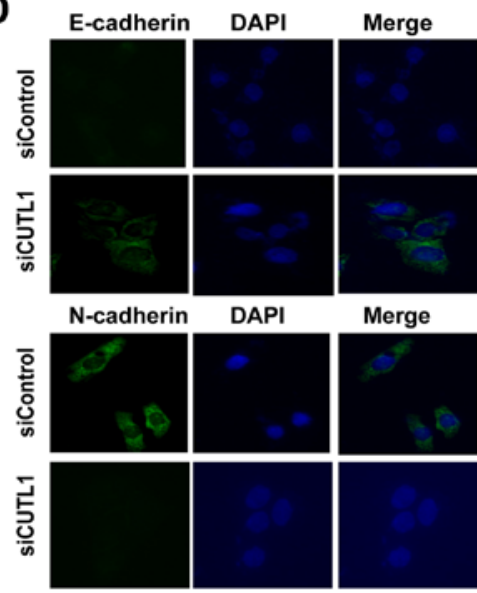

Figure 3. Effects of CUTL1 on EMT in NSCLC cell lines. (A) Western blot analysis of CUTL1, E-cadherin, N-cadherin and Snail expression in H322 (left panel) and H292 (right panel) cells. (B) Western blot analysis of CUTL1, E-cadherin, N-cadherin and Snail expression in A549 cells. (C) siCUTL1 A549 and (D) Calu1 cells were subjected to immunofluoresence staining with E-cadherin or N-cadherin antibody. DAPI was used to visualize the nuclei.

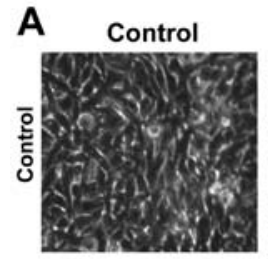

SB431542
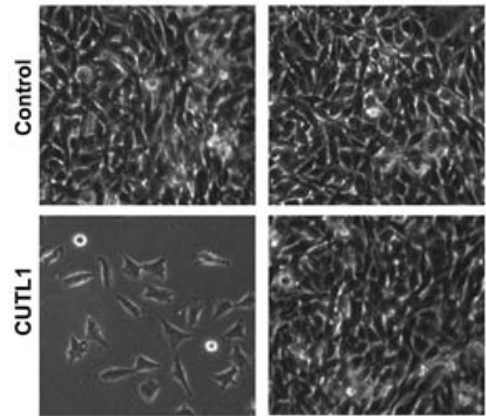

\section{C}

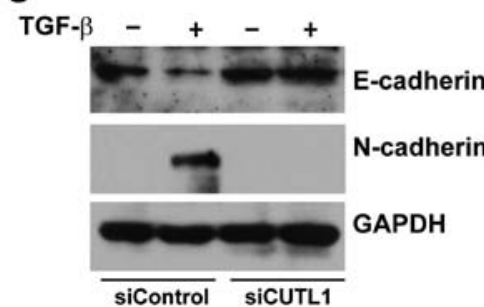

B SB431542

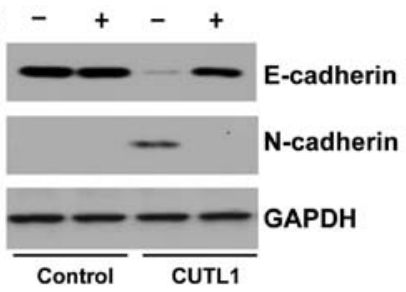

SB431542

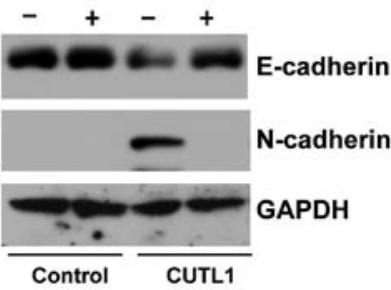

TGF- $\beta$

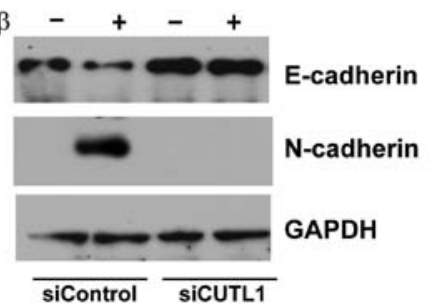

Figure 4. CUTL1 induces EMT through the TGF- $\beta$ signaling pathway. (A) Effect of SB431542 on morphology of siControl and siCUTL1 A549 cells. (B) Western blot analysis was used to determine the effect of SB431542 on E-cadherin and N-cadherin expression in CUTL1-overexpressing H322 (upper panel) and H292 (lower panel) cells. GAPDH was used as control. (C) Western blot analysis was used to determine the E-cadherin and N-cadherin expression in A549 (left panel) and Calu1 (right panel) cells following CUTL1 depletion. GAPDH was used as control. 

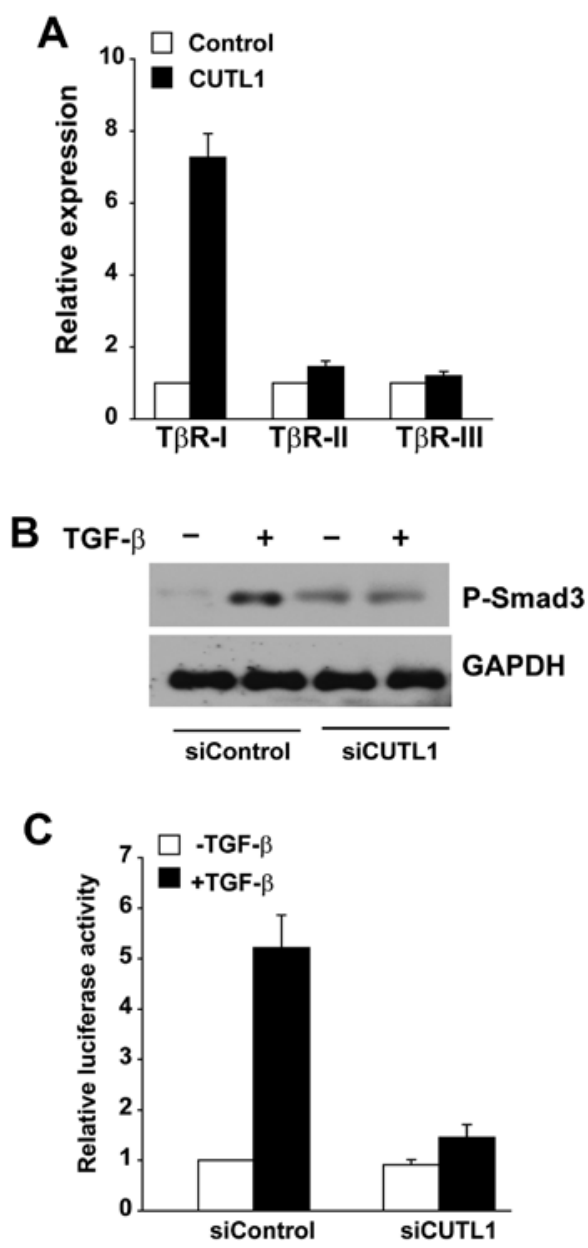

Figure 5. CUTL1 promotes T $\beta$ R-I expression to positively regulate the TGF- $\beta$ signaling pathway. (A) qRT-PCR was used to determine the effect of ectopic CUTL1 expression on T $\beta$ R-I, T $\beta$ R-II and T $\beta$ R-III expression in H322 cells. (B) A549 cells transfected with siControl and siCUTL1 were stimulated with $2 \mathrm{ng} / \mathrm{ml}$ of TGF- $\beta 1$ for $1 \mathrm{~h}$ and then harvested. The expression levels of p-Smad3 proteins were examined by western blotting. (C) A549 cells were transfected with the reporter p3TP-Lux plasmid in the absence and presence of siCUTL1. The cells were harvested at $36 \mathrm{~h}$ after transfection and assayed for luciferase activity. The data are the means \pm SD.

be an important mediator of tumor cell motility and invasiveness in many types of tumors. The molecular mechanism for promoting migration by CUTL1 has been reported. For instance, CUTL1 accelerates tumor cell migration via decreasing proteasome-mediated Src degradation (9), and CUTL1 uses WNT5A as an important target to promote invasiveness and tumor progression in pancreatic cancer (11). However, it is unknown whether CUTL1 plays a key role in the EMT process $(18,19)$. In the present study, we provide evidence that CUTL1 induces EMT in NSCLC. CUTL1 may facilitate tumor progression, and targeting its expression will be a promising strategy for cancer therapy (6). In this study, we detected the expression of CUTL1 in NSCLC tissues. The results showed that CUTL1 was overexpressed in human NSCLC. Additionally, the effects of CUTL1 on the migration and invasion of lung cancer cells were studied, and we found that CUTL1 could enhance NSCLC cellular migration and invasion. These data suggest that CUTL1 is an oncogene and provide a strategy for cancer treatment.
EMT plays a pivotal role in cancer metastasis. During this process, tumor cells derived from epithelial cells lose their epithelial features and acquire mesenchymal features to escape from the primary tissue and invade the surrounding stroma $(12,20,21)$. E-cadherin is a cell adhesion molecule involved in maintaining the epithelial phenotype. The loss of E-cadherin often results in a loss of epithelial morphology (22). Notably, the decreased expression of E-cadherin is often accompanied by upregulation of the expression of $\mathrm{N}$-cadherin, which promotes tumor cell invasiveness (23). Snail is another important EMT marker that is well known to enhance tumor invasion (24). In this study, CUTL1 was shown to decrease the expression of E-cadherin and increase the expression of $\mathrm{N}$-cadherin and Snail. These results suggest that CUTL1 is able to induce EMT. Moreover, the migration and invasion assays further confirm that CUTL1 promotes cell migration and invasion in vitro.

CUTL1 (CCAAT displacement protein 1) belongs to the homeodomain transcription factor family. Because the transcription factor CUTL1 contains the DNA-binding domain, we speculated that CUTL1 interacts with T $\beta$ R-I promoter through its DNA-binding site, which facilitates CUTL promoting T $\beta$ R-I expression. We show that CUTL1 directly facilitates T $\beta$ R-I expression by activating T $\beta$ R-I gene transcription, indicating that activation of the TGF- $\beta$ signaling network may be related to CUTL1-induced EMT. This is clearly supported by the observation that CUTL1-induced EMT in epithelial-like lung cancer cells was reversed by the T $\beta R-I$ inhibitor SB431542. It is well-known that TGF- $\beta$ functions as a characterized inducer of EMT during cancer progression and metastasis (25). TGF- $\beta$ signaling is activated through a heteromeric complex of type I and type II transmembrane serine/threonine kinase receptors. Then, the receptor complex results in the activation of Smad2 and Smad3. Next, phosphorylated Smad2/3 and Smad4 translocate to the nucleus, where they cooperate with transcription factors such as Snail to inhibit the expression of epithelial markers and promote the expression of mesenchymal markers at the mRNA level $(26,27)$.

Tumor cells induced by TGF- $\beta$ become invasive concomitant with the loss of epithelial characteristics (28). TGF- $\beta$-induced EMT leads to cadherin switching, i.e., the loss of E-cadherin and the increased presence of N-cadherin, which results in cells becoming more motile and invasive $(29,30)$. Tumor cells often secrete active TGF- $\beta$; therefore, the measurement of the serum concentration of TGF- $\beta$ has been a complementary diagnostic method in lung cancer detection (31). Our experiments showed that CUTL1 induced EMT in NSCLC. All of these studies indicate that CUTL1 is a potential target for anti-lung cancer therapy.

\section{Acknowledgements}

The present study was supported by the National Natural Science Foundation of China (81172818; 81172215).

\section{References}

1. Shtivelman E, Hensing T, Simon GR, Dennis PA, Otterson GA, Bueno R and Salgia R: Molecular pathways and therapeutic targets in lung cancer. Oncotarget 5: 1392-1433, 2014. 
2. Reck M,Heigener DF, Mok T, Soria JC and Rabe KF: Management of non-small-cell lung cancer: Recent developments. Lancet 382: 709-719, 2013.

3. Hsiao SH, Chung CL, Chou YT, Lee HL, Lin SE and Liu HE: Identification of subgroup patients with stage IIIB/IV non-small cell lung cancer at higher risk for brain metastases. Lung Cancer 82: 319-323, 2013.

4. Nepveu A: Role of the multifunctional CDP/Cut/Cux homeodomain transcription factor in regulating differentiation, cell growth and development. Gene 270: 1-15, 2001.

5. Sansregret L and Nepveu A: The multiple roles of CUX1: Insights from mouse models and cell-based assays. Gene 412: 84-94, 2008

6. Liu KC, Lin BS, Zhao M, Wang KY and Lan XP: Cutl1: A potential target for cancer therapy. Cell Signal 25: 349-354, 2013.

7. Truscott M, Raynal L, Premdas P, Goulet B, Leduy L, Bérubé G and Nepveu A: CDP/Cux stimulates transcription from the DNA polymerase alpha gene promoter. Mol Cell Biol 23: 3013-3028, 2003.

8. van Gurp MF, Pratap J, Luong M, Javed A, Hoffmann H, Giordano A, Stein JL, Neufeld EJ, Lian JB, Stein GS, et al: The CCAAT displacement protein/cut homeodomain protein represses osteocalcin gene transcription and forms complexes with the retinoblastoma protein-related protein p107 and cyclin A. Cancer Res 59: 5980-5988, 1999.

9. Aleksic T, Bechtel M, Krndija D, von Wichert G, Knobel B, Giehl K, Gress TM and Michl P: CUTL1 promotes tumor cell migration by decreasing proteasome-mediated Src degradation. Oncogene 26: 5939-5949, 2007.

10. Fan X, Wang H, Zhou J, Wang S, Zhang X, Li T, Nie Y and Liu B: The transcription factor CUTL1 is associated with proliferation and prognosis in malignant melanoma. Melanoma Res 24 198-206, 2014.

11. Ripka S, König A, Buchholz M, Wagner M, Sipos B, Klöppel G, Downward J, Gress T and Michl P: WNT5A - target of CUTL1 and potent modulator of tumor cell migration and invasion in pancreatic cancer. Carcinogenesis 28: 1178-1187, 2007.

12. Zheng $\mathrm{H}$ and Kang Y: Multilayer control of the EMT master regulators. Oncogene 33: 1755-1763, 2014.

13. Tsai JH and Yang J: Epithelial-mesenchymal plasticity in carcinoma metastasis. Genes Dev 27: 2192-2206, 2013.

14. Foroni C, Broggini M, Generali D and Damia G: Epithelialmesenchymal transition and breast cancer: Role, molecular mechanisms and clinical impact. Cancer Treat Rev 38: 689-697, 2012.

15. Lamouille $\mathrm{S}, \mathrm{Xu} \mathrm{J}$ and Derynck R: Molecular mechanisms of epithelial-mesenchymal transition. Nat Rev Mol Cell Biol 15: 178-196, 2014.

16. Fabregat I, Fernando J, Mainez J and Sancho P: TGF-beta signaling in cancer treatment. Curr Pharm Des 20: 2934-2947, 2014.
17. Hong S, Noh H, Teng Y, Shao J, Rehmani H, Ding HF, Dong Z $\mathrm{Su} \mathrm{SB}$, Shi H, Kim J, et al: SHOX2 is a direct miR-375 target and a novel epithelial-to-mesenchymal transition inducer in breast cancer cells. Neoplasia 16: 279-90.e1, 5, 2014

18. Michl P and Downward J: CUTL1: A key mediator of TGFbetainduced tumor invasion. Cell Cycle 5: 132-134, 2006.

19. Michl P, Ramjaun AR, Pardo OE, Warne PH, Wagner M, Poulsom R, D'Arrigo C, Ryder K, Menke A, Gress T, et al: CUTL1 is a target of TGF(beta) signaling that enhances cancer cell motility and invasiveness. Cancer Cell 7: 521-532, 2005.

20. Tiwari N, Gheldof A, Tatari M and Christofori G: EMT as the ultimate survival mechanism of cancer cells. Semin Cancer Biol 22: 194-207, 2012.

21. Christiansen JJ and Rajasekaran AK: Reassessing epithelial to mesenchymal transition as a prerequisite for carcinoma invasion and metastasis. Cancer Res 66: 8319-8326, 2006.

22. Nagathihalli NS, Massion PP, Gonzalez AL, Lu P and Datta PK: Smoking induces epithelial-to-mesenchymal transition in non-small cell lung cancer through HDAC-mediated downregulation of E-cadherin. Mol Cancer Ther 11: 2362-2372, 2012.

23. Zhang X, Liu G, Kang Y, Dong Z, Qian Q and Ma X: N-cadherin expression is associated with acquisition of EMT phenotype and with enhanced invasion in erlotinib-resistant lung cancer cell lines. PLoS One 8: e57692, 2013.

24. Zheng P, Meng HM, Gao WZ, Chen L, Liu XH, Xiao ZQ, Liu YX, Sui HM, Zhou J, Liu YH, et al: Snail as a key regulator of PRL-3 gene in colorectal cancer. Cancer Biol Ther 12: 742-749, 2011.

25. Moustakas A and Heldin $\mathrm{CH}$ : Signaling networks guiding epithelial-mesenchymal transitions during embryogenesis and cancer progression. Cancer Sci 98: 1512-1520, 2007.

26. Feng XH and Derynck R: Specificity and versatility in tgf-beta signaling through Smads. Annu Rev Cell Dev Biol 21: 659-693, 2005.

27. Shi Y and Massagué J: Mechanisms of TGF-beta signaling from cell membrane to the nucleus. Cell 113: 685-700, 2003.

28. Thiery JP and Sleeman JP: Complex networks orchestrate epithelial-mesenchymal transitions. Nat Rev Mol Cell Biol 7: 131-142, 2006.

29. Cavallaro $\mathrm{U}$ and Christofori G: Cell adhesion and signalling by cadherins and Ig-CAMs in cancer. Nat Rev Cancer 4: 118-132, 2004.

30. Shirakihara T, Saitoh M and Miyazono K: Differential regulation of epithelial and mesenchymal markers by deltaEF1 proteins in epithelial mesenchymal transition induced by TGF-beta. Mol Biol Cell 18: 3533-3544, 2007.

31. González-Santiago AE, Mendoza-Topete LA, Sánchez-Llamas F, Troyo-Sanromán R and Gurrola-Díaz CM: TGF- $\beta 1$ serum concentration as a complementary diagnostic biomarker of lung cancer: Establishment of a cut-point value. J Clin Lab Anal 25: 238-243, 2011. 